14. Coyne JD. Necrobiotic palisading granulomas associated with breast carcinoma. J Clin Pathol 2005; 58: 1290-3.

15. Barr RJ, Stegman SJ. Delayed skin test reaction to injectable collagen implant (Zyderm). The histopathologic comparative study. J Am Acad Dermatol 1984; 10: 652-8.

DOI: https://doi.org/10.1016/j.pathol.2018.05.012

\section{Atypical finding of meningothelial- like inclusions in cervical lymph nodes}

Sir,

Incidental findings of benign spindle-shaped cells with oval to indented spindle nuclei inclusions have been described predominantly in lung cancer cases and given the name minute pulmonary meningothelial-like nodules or MPMN. ${ }^{1-3}$ It was proposed by Gaffey et al. in $1988^{1}$ that the morphological characteristics of a meningothelial-like nodule closely resemble meningothelial cells. This relationship has been supported by immunohistochemical (IHC) analysis which reveals the strong reactivity of MPMN to epithelial membrane antigen (EMA) and the filament protein vimentin. In contrast, other papers have reported 'meningothelial-like whorls' from tissue outside of the lung, ${ }^{2-4}$ describing positive staining for myosin, alpha smooth muscle actin ( $\alpha$-SMA) and negative for EMA, suggesting muscle origin. Due to the inconsistent IHC results, the origin of these benign inclusions is unclear. It is yet to be determined if anatomical location of the inclusion reflects the IHC profile. Herein, we report a unique case of benign meningothelial inclusions in cervical lymph nodes, which has not been reported previously to the authors' knowledge.

A 65-year-old Caucasian male presented for a total laryngectomy and neck dissection for squamous cell carcinoma (SCC). Neck dissection included the removal of 49 lymph nodes of which zero contained SCC. Microscopically, six of
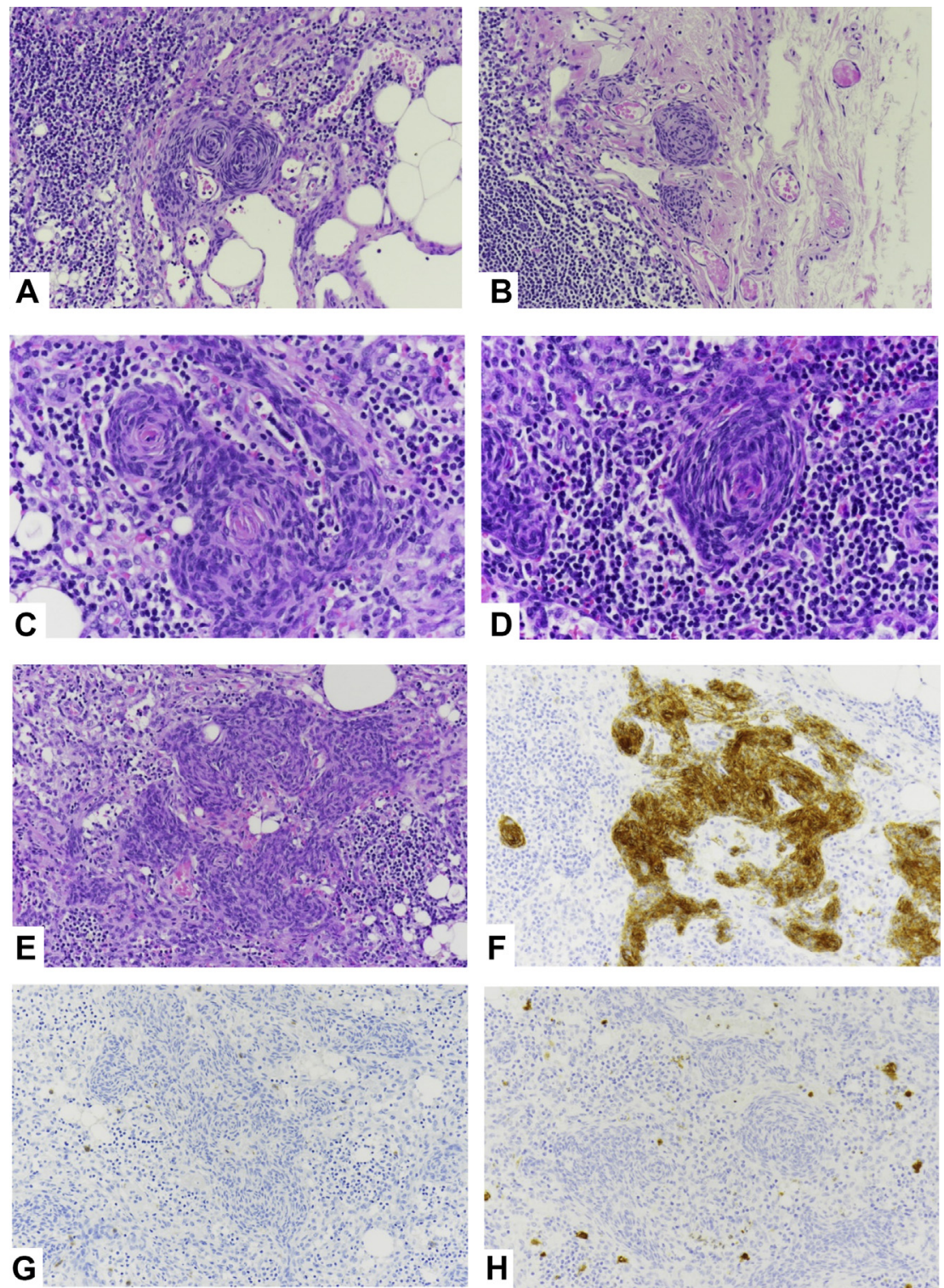

Fig. 1 (A-D) Lymph node biopsy with meningothelial-like inclusions exhibiting distinct spindle-shaped cells with oval to indented spindle nuclei (A,B: H\&E, medium power; C,D: H\&E, high power). (E-H) All show the same area, medium power: (E) H\&E; (F) positive IHC staining for EMA; (G) negative IHC staining for AE1/AE3; and $(\mathrm{H})$ negative IHC staining for $\mathrm{S} 100$ 
the 49 nodes contained benign meningothelial-like inclusions, characterised by spindle-shaped cells with indented spindle nuclei (Fig. 1A-E).

Immunohistochemistry was performed on the nodes which were positive for CD99 (cytoplasmic), vimentin and EMA (Fig. 1F) and negative for progesterone receptor (PR), D2-40, AE1/AE3 (Fig. 1G), p40, S100 (Fig. 1H), calretinin, TDT, CD68, MelanA, chromogranin, synaptophysin, Sox10, myosin and $\alpha$-SMA. Electron microscopy was performed on back-processed tissue and therefore was of low quality. However, it did appear to show interdigitating extensions similar to those described by Niho et $a l^{5}$ as an 'organoid pattern with nest cells' (Fig. 2).

This is the first reported case of meningothelial-like in clusions from lymph nodes in the neck. The inclusions from the six lymph nodes consisted of the previously described morphological elements. However, this emphasises the fact that varied IHC results reported in the literature from sites other than the lung, may pose a challenge when attempting to confirm a consistent histochemical origin.

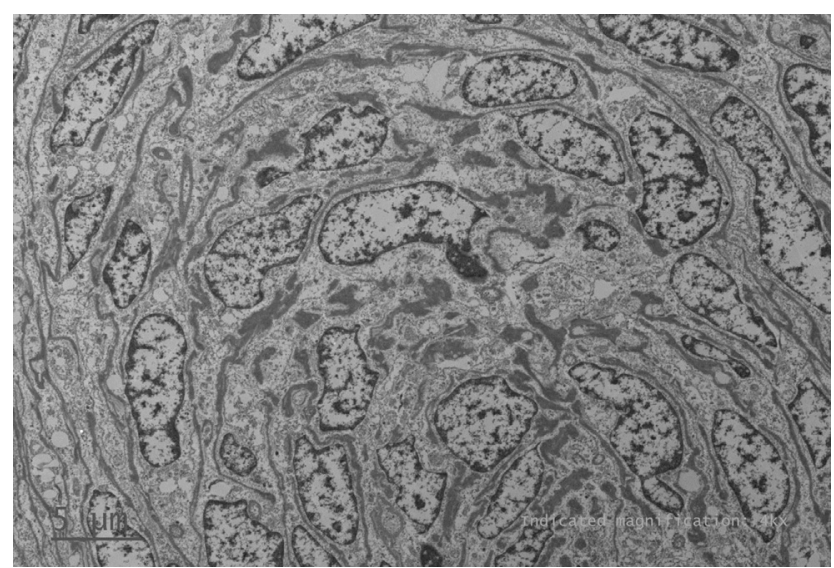

Fig. 2 Electron micrograph showing interdigitating extensions from a meningothelial-like inclusion. Euchromatin predominates in the nuclei and long branching processes stem from the cell bodies, forming a spindle shape $(\times 4000)$.
A review of meningothelial-like inclusion case studies was performed in January 2018 using databases from Scopus, PubMed and ProQuest containing the search terms 'meningothelial-like nodules' OR 'meningothelial whorls' OR 'chemodectoma' in any field (title, subject, abstract). A manual review of the identified articles was performed to identify any duplications. Data such as the first author, publication year, country of publication, patient sex and age, location of the tissue, IHC results and other diagnostic measures were extracted for further evaluation. A total of 1167 papers were published between 1951 and 2018 but after evaluation for duplications and exclusions (English language, search term in title, journal article only, human-only cases), 92 were chosen for detailed analysis. There were 13 results for 'meningothelial-like nodules' from 1999-2017; five results for 'meningothelial whorls/inclusions' from 19982010; and 74 results for 'chemodectoma' from 1951-2017. Case studies which reported chemodectomas only were excluded from the review.

When meningothelial-like nodules of the lung were first described (before the availability of diagnostic immunohistochemistry), they were believed to be 'chemodectomas' (now known as paraganglioma). Paragangliomas are consistently positive for neuroendocrine markers in contrast to meningothelial-like nodules. ${ }^{6,7}$ Suggestions of muscle origin have been supported ${ }^{2-4}$ from the IHC profile but variable results have been reported by others. ${ }^{5,8}$ If reported, the common findings for IHC results include weak to strong positive staining for: vimentin, EMA, PR, CD34, CD68, CD56, CD10, S100, p53, $\alpha$-SMA, cytokeratin 5, 6, 8, 19 and NSE. Negative to diffuse staining occurs for: MDM-2, CD21, CD31, CD34, CD35, CD57, cytokeratin AE1/AE3, desmin, INSM1, myosin, alpha inhibin and 'neuroendocrine, vascular, melanocytic and estrogen' markers.

Predominant demographic characteristics for meningothelial-like inclusions include an increased female to male ratio, with an average age of 60 years. Some reported cases have come from incidental findings from examination of breast, ${ }^{7}$ liposarcoma ${ }^{3,4,9}$ and endometrial tumours. ${ }^{10}$ Table 1 provides a summary of meningothelial-like

Table 1 Overview of studies reporting meningothelial-like inclusions outside of the lung between 1998-2017

\begin{tabular}{|c|c|c|c|c|}
\hline Author/reference & Country of origin & Anatomical site/Lesion & IHC positive & IHC negative \\
\hline Santi $(2010)^{12}$ & Australia & $\begin{array}{l}1 \times \text { retroperitoneal paravertebral } \\
\text { mass, cellular schwannoma }\end{array}$ & S100 & MDM-2, EMA \\
\hline Wang $(2008)^{11}$ & China & $\begin{array}{l}1 \times \text { skin-dermatofibrosarcoma } \\
\text { protuberans (adipose tissue) }\end{array}$ & CD34 & $\begin{array}{l}\text { EMA, S100, cytokeratin AE1/AE3, } \\
\text { alpha smooth muscle actin, } \\
\text { muscle-specific actin (HHF35), } \\
\text { desmin and HMB45 }\end{array}$ \\
\hline $\operatorname{Kim}(2003)^{3}$ & Korea & $\begin{array}{l}1 \times \text { anterior mediastinum (adipose tissue) } \\
2 \times \text { retroperitoneum (adipose tissue, } \\
\text { rectus muscle); } 1 \times \text { scrotum (lipoma) }\end{array}$ & $\begin{array}{l}\text { Vimentin, alpha smooth } \\
\text { muscle actin, CD10, } \\
\text { CD56, CD99, factor } \\
\text { XIII, low affinity nerve } \\
\text { growth factor receptor }\end{array}$ & $\begin{array}{l}\text { CD21, CD31, CD34, CD35, } \\
\text { CD57, CD68, Cytokeratin } \\
\text { AE1/AE3, desmin, } \\
\text { E-cadherin, EMA, S100 } \\
\text { (diffuse p53 and Ki-67) }\end{array}$ \\
\hline $\begin{array}{l}\text { Fanburg-Smith } \\
\qquad(1998)^{4}\end{array}$ & USA & $17 \times$ liposarcoma: retroperitoneal tumours & $\begin{array}{l}\text { Alpha smooth muscle } \\
\text { actin, p53, osteocalcin }\end{array}$ & $\begin{array}{l}\text { EMA, CD21, CD35, CD34, } \\
\quad \text { CD31 }\end{array}$ \\
\hline Kfoury $(2012)^{7}$ & Korea & $1 \times$ breast (adipose and breast stroma) & $\begin{array}{l}\text { Vimentin, E-cadherin, } \\
\text { EMA, progesterone }\end{array}$ & $\begin{array}{l}\text { Neuroendocrine, vascular, } \\
\text { melanocytic, oestrogen markers }\end{array}$ \\
\hline Zamecnik $(2007)^{10}$ & Czech Republic & $2 \times$ endometrial (smooth muscle) & $\begin{array}{l}\text { Alpha smooth muscle } \\
\text { actin, CD10, calponin }\end{array}$ & $\begin{array}{l}\text { Desmin, h-caldesmon, EMA, } \\
\text { cytokeratin AE1/AE3 and } \\
\text { alpha inhibin }\end{array}$ \\
\hline
\end{tabular}


inclusions reported from 1998-2017 outside of the lung. The literature has not previously reported a case of meningothelial-like inclusions from lymph nodes in the neck. Findings from this report suggest: (1) although rare, benign meningothelial-like inclusions can be identified in tissue outside of the lung; (2) the IHC profile of meningothelial-like inclusions is inconsistent and shows variability in tissue sites other than the lung.

It is postulated that the benign spindle shaped cells located in the lymph nodes in this case are mesenchymal in origin. Some mesenchymal cells can retain plasticity in the adult and are upregulated to differentiate into diverse cell types when needed, such as in the case of tissue repair/remodelling in response to an injury or a tumour. Mesenchymal cells have the ability to migrate through tissue and are known to produce cytokines and growth factors that influence the differentiation of surrounding normal cells in the body such as epithelium, connective tissue and muscle.

This may explain why the IHC profile of meningotheliallike inclusions is inconsistent and there is variability in different tissue sites. For example, meningothelial-like inclusions from endometrial tissue have positive IHC for smooth muscle markers, ${ }^{10}$ whereas the same IHC markers will be negative for meningothelial-like inclusions in adipose tissue. ${ }^{11}$ In this case the IHC characteristics suggest reactive cells of normal tissue (EMA positive), that contain intermediate filament protein (vimentin) involved in membranous $\mathrm{T}$ cell adhesion and leukocyte migration (CD99 positive) because the site of origin is lymphoid tissue. These benign meningothelial-like inclusions do not suggest muscle (myosin and $\alpha$-SMA negative), neural (synaptophysin and Sox10 negative), or neuroendocrine (chromogranin negative) origin.

Conflicts of interest and sources of funding: The authors state that there are no conflicts of interest to disclose.

\section{Rebecca Donkin ${ }^{1}$, Andrew Dettrick ${ }^{2}$, Penelope Wyche $^{2}$, Sarah Grigg ${ }^{2}$}

${ }^{1}$ University of the Sunshine Coast, Sippy Downs, Qld, Australia; ${ }^{2}$ Sunshine Coast University Hospital, Qld, Australia

Contact Dr Andrew Dettrick.

E-mail: andrew.dettrick@health.qld.gov.au

1. Gaffey MJ, Mills SE, Askin FB. Minute pulmonary meningothelial-like nodules. A clinicopathologic study of so-called minute pulmonary chemodectoma. Am J Surg Pathol 1988; 12: 167-75.

2. Torikata C, Mukai M. So-called minute chemodectoma of the lung. An electron microscopic and immunohistochemical study. Virchows Arch A Pathol Anat Histopathol 1990; 417: 113-8.

3. Kim S, Choi Y, Kim H, Yang W. Liposarcoma with meningothelial-like whorls. Report of four cases showing diverse histologic findings and behavior. Yonsei Med J 2003; 44: 392-400.

4. Fanburg-Smith J, Miettinen M. Liposarcoma with meningothelial-like whorls: a study of 17 cases of a distinctive histological pattern associated with dedifferentiated liposarcoma. Histopathology 1998; 33: 414-24.

5. Niho S, Yokose T, Nishiwaki Y, Mukai K. Immunohistochemical and clonal analysis of minute pulmonary meningothelial-like nodules. Hum Pathol 1999; 30: 425-9.

6. Dermawan JK, Mukhopadhyay S. Insulinoma-associated protein (INSM1) differentiates carcinoid tumorlets of the lung from pulmonary meningothelial-like nodules. Histopathology 2017; 72: 1067-9.
7. Kfoury H, Arafah MA, Arafah MM, Alnassar S, Hajjar W. Mimicry of minute pulmonary meningothelial-like nodules to metastatic deposits in a patient with infiltrating lobular carcinoma: a case report and review of the literature. Korean J Pathol 2012; 46: 87.

8. Ionescu D, Omalu B, Finkelstein SD, et al. Pulmonary meningotheliallike nodules - a genotypic comparison with meningiomas. Lab Invest 2003; 83: 308A.

9. Matsumoto T, Fukunaga M, Fujii H, et al. Inguinal dedifferentiated liposarcoma with meningothelial-like whorls and metaplastic bone formation. Histopathology 2005; 46: 594.

10. Zamecnik M, Sultani K. Meningothelial-like nodules: additional pattern of myoid differentiation in endometrial stromal tumors. Pathol Int 2007; 57: $632-3$.

11. Wang J, Yang W. Pigmented dermatofibrosarcoma protuberans with prominent meningothelial-like whorls. J Cutan Pathol 2008; 35 (Suppl 1): 65 .

12. Santi R, Franchi A, Veltri M, Valeri A, Nesi G. Meningothelial-like whorls in a retroperitoneal cellular schwannoma: potential diagnostic pitfall. Pathol Int 2010; 60: 62-3.

DOI: https://doi.org/10.1016/j.pathol.2018.06.003

\section{Prostatic adenocarcinoma with aberrant diffuse expression of high molecular weight cytokeratin}

Sir,

Immunohistochemistry for high molecular weight cytokeratin (HMWCK) and p63 as basal cell markers has been widely used for the diagnosis of limited prostatic adenocarcinoma that lacks basal cells with rare exceptions. ${ }^{1}$ While aberrant diffuse expression of p63 can be uncommonly seen in prostate cancer, ${ }^{2,3}$ advantages in using HMWCK immunohistochemistry include no such diffuse HMWCK-positive prostate cancer reported. ${ }^{1}$ Instead, several studies have demonstrated focal HMWCK expression in rare cases of primary prostatic adenocarcinoma. ${ }^{4-7}$ We present a case of prostate cancer showing diffuse immunoreactivity for HMWCK.

A 93-year-old male with a history of benign prostatic hyperplasia presented to the urology clinic with gross haematuria. His serum prostate-specific antigen (PSA) was $34.2 \mathrm{ng}$ / $\mathrm{mL}$. On digital rectal examination, he was found to have an enlarged prostate without nodularity or induration. Cystoscopy identified a large bladder mass, estimated at $5 \mathrm{~cm}$, along the right lateral wall extending from the bladder neck. The mass was then transurethrally resected in its entirety.

The surgical specimen histologically showed poorly differentiated adenocarcinoma (Fig. 1A,B). Immunohistochemical studies revealed that the tumour cells were positive for prostate markers, including Nkx3.1 (Fig. 1C,E), PSA (Fig. 1D,E), P501S prostein (Fig. 1E), and prostatic acid phosphatase (Fig. 1F), and negative for GATA3 (clone L50823). PIN4 multiplex staining (Fig. 1G,H) further showed negativity of p63 (clone 13H4) and focal positivity of P504S $\alpha$-methylacyl-CoA racemase (clone $4 \mathrm{~A} 4$ ) as well as diffuse moderate to strong membranous positivity of HMWCK (clone $34 \beta E 12)$ throughout the tumour. Positive controls for all these stains and negative tissue elements were both evaluated and were adequate for diagnosis. Based on the morphology and immunoprofile, a diagnosis of prostatic adenocarcinoma, Gleason score $4+5=9$ [International Society of Urological Pathology Grade 5 (otherwise known as 